\title{
Problematiche diagnostiche nelle infezioni sostenute da Legionella pneumophila
}

\author{
Manuela Pegoraro, Laura Maccacaro, Roberta Fontana
}

Servizio di Microbiologia, Immunologia e Virologia, Ospedale Civile Maggiore, Azienda Ospedaliera di Verona e Università di Verona

\section{Problems in diagnosis of Legionella pneumophila infections}

Key words: Urine antigen detection test, Colture isolation, Serologic diagnosis

\section{SUMMARY}

The introduction of the research of Legionella antigen in urine has significantly speeded up the diagnosis of Legionella pneumophila infections but has decreased the number of respiratory samples submitted for coltural examination. In $200 \mathrm{I}$ respiratory samples were submitted to our laboratory only from $7 \%$ of patients for which antigen detection in urine was requested. Such a percentage decreased to $4 \%$ in 2002 and to $2.5 \%$ in 2003 . For 2 of 14 patients tested in 2002, legionellosis diagnosis could not be confirmed since urine antigen detection provided weak positive values and either respiratory samples for direct isolation of L. pneumophila or blood samples for specific serology tests were lacking. We believe that even if urine antigen detection test is a very useful quick test, it cannot be considered as the only diagnostic tool in Legionella spp. infections.

\section{INTRODUZIONE}

Legionella pneumophila è sicuramente la più importante delle 48 specie comprese nel genere Legionella, sia dal punto di vista clinico sia epidemiologico, anche se almeno la metà di quelle finora identificate sono state associate a patologie nell'uomo $(3,7)$. Veicolati da aerosol, questi batteri passano dal loro habitat naturale, rappresentato da ambienti acquatici naturali e artificiali, agli alveoli polmonari, dove innescano un processo infiammatorio essudativo che si rende manifesto con un quadro di polmonite purulenta a focolai isolati o confluenti, spesso bilaterali e accompagnati da un marcato versamento pleurico (16). Solo per le infezioni sostenute da L. longbeachae è stata finora individuata una sorgente diversa dall'acqua rappresentata dal terreno di giardinaggio (10). Data l'ubiquitarietà del microrganismo è evidente che lo sviluppo della malattia è legato anche a fattori di rischio predisponenti che sono stati identificati nel tabagismo, nell'alcolismo, nella presenza di malattie croniche polmonari (ma non soltanto polmonari) e in tutti gli stati di immunocompromissione, sia acquisita sia iatrogena $(13,14,16)$. Secondo quanto riportato in letteratura, Legionella spp. sarebbe responsabile dell' $1-5 \%$ delle polmoniti comunitarie e del 5-15\% di quelle nosocomiali $(6,14,17)$. Nonostante il numero di notifiche riportate dall'Istituto Superiore di Sanità (ISS) nel "Rapporto annuale sulla legionellosi" sia significativamente aumentato in Italia negli ultimi anni (585 casi nel 2002 rispetto ai 325 del 2001), si ritiene che la malattia sia ancora sottostimata sia per difficoltà diagnostiche sia per scarsa attenzio- ne nelle procedure di notifica. Infatti lo stesso documento attribuiva al Veneto 10 casi di legionellosi nel 2001 mentre nella sola provincia di Verona ne sono stati diagnosticati $8(1,4)$.

Nella sua espressione tipica, la malattia del legionario si manifesta con i sintomi di una polmonite (febbre elevata ad esordio brusco, tosse non produttiva, dispnea e dolori toracici) a cui possono associarsi alterazioni gastrointestinali (nausea, vomito, diarrea) e neurologiche (cefalea, confusione mentale) $(14,16)$.

Le difficoltà diagnostiche legate soprattutto alla necessità di tempi prolungati di incubazione per l'isolamento colturale e alla interferenza da parte di altri batteri a più rapida crescita e comunemente presenti nelle alte vie respiratorie, sono state in parte superate dall'introduzione della ricerca dell'antigene specifico nelle urine. Grazie alla semplicità di esecuzione e alla rapidità con cui permette di ottenere il risultato, questo test è diventato 1'approccio più largamente utilizzato nella diagnosi di infezioni sostenute da L. pneumophila $(11,14,15)$. Nelle stesse linee guida dell'ISS il risultato ottenuto con questo tipo di indagine viene ritenuto sufficiente a fornire il criterio microbiologico di certezza nella definizione di caso anche se l'importanza della ricerca colturale viene comunque chiaramente sottolineata (9).

Il nostro studio riporta i risultati di tre anni di attività diagnostica delle infezioni sostenute da $L$. pneumophila, nel territorio di afferenza del Servizio di Microbiologia dell'Ospedale Civile Maggiore (O.C.M.) di Verona, ottenuti con l'utilizzo combinato di diversi metodi di indagine. Nel 
sottolineare le difficoltà interpretative e diagnostiche emerse con la diminuzione della richiesta di indagini colturali e delle conferme sierologiche associate all'introduzione del test urinario, si vuole ribadire la necessità di non limitare l'approccio diagnostico al saggio dell'antigenuria ma di affiancarlo sempre alla ricerca colturale del microrganismo nei materiali idonei e al monitoraggio della risposta anticorpale.

\section{MATERIALI E METODI}

Nel periodo 2001-2003 sono stati analizzati 474 campioni di urina, corrispondenti a 408 pazienti, inviati per la ricerca dell'antigene di L. pneumophila. Nel 2001 sono pervenuti 79 campioni corrispondenti a 72 pazienti, nel 2002 i campioni sono stati 214 (relativi a 180 pazienti) e nel 2003 infine, 181 campioni corrispondenti a 156 pazienti. Il saggio, basato su un metodo immunoenzimatico, è stato eseguito con il kit "Legionella Urin Antigen" (Biotest, Dreieich - Germania) su campioni freschi, o mantenuti a $4^{\circ} \mathrm{C}$ per 24 ore al massimo e centrifugati (1000 rpm per $10 \mathrm{~min}$ ). I campioni sono stati interpretati come positivi per valori di assorbanza superiori a 0.4 , negativi per valori inferiori a $0.2 \mathrm{e}$ dubbi per valori compresi tra 0.2 e 0.4 .

I materiali respiratori sottoposti ad esame colturale sono stati soltanto 16 e così ripartiti: 5 nel 2001, 7 nel 2002 e 4 nel 2003. I campioni, 15 fra broncoaspirati e broncolavaggi e 1 escreato, sono stati concentrati mediante centrifugazione $(3400 \mathrm{~g}$ per $20 \mathrm{~min}$ ); parte del sedimento è stato quindi seminato tal quale su terreno selettivo (Buffered Charcoal Yeast Extract - Polimixina, Anisomocina, Cefamandolo; Oxoid, Basingstoke, Hampshire) e su agar sangue, parte è stata decontaminata tramite contatto con soluzione $\mathrm{HCl}-\mathrm{KCl}$ a pH $2.2(5.3 \mathrm{ml}$ di $\mathrm{HCl} 0.2 \mathrm{~N}, 25$ $\mathrm{ml}$ di $\mathrm{KCl} 0.2 \mathrm{~N}$ in $100 \mathrm{ml}$ di acqua distillata) in rapporto 1:10 per 4 min e quindi seminata sugli stessi terreni. Le piastre sono state incubate in microaerofilia a $37^{\circ} \mathrm{C}$ e osservate giornalmente per 10 giorni. Le colonie sospette sono state identificate tramite immunofluorescenza utilizzando anticorpi tipospecifici (Zeus Scientific, Inc. Raritan, NJ).

L'indagine sierologica è stata eseguita su 2933 campioni di sangue (corrispondenti a 2383 pazienti) tramite immunofluorescenza indiretta utilizzando un kit che permette di valutare la risposta anticorpale nei confronti di 15 sierotipi di L. pneumophila, suddivisi in 4 gruppi (Euroimmun, Lubecca - Germania). L'elevato numero di campioni di sangue analizzati deriva dal fatto che la ricerca di anticorpi anti-L. pneumophila viene sempre eseguita, nel nostro Laboratorio, nell'ambito della diagnosi sierologi- ca delle polmoniti atipiche anche in assenza di richiesta mirata.

\section{RISULTATI}

Nei tre anni presi in considerazione 2570 pazienti sono stati sottoposti a test per la diagnosi di infezione da L. pneumophila. Nelle tabelle 1, 2 e 3 sono riportati i risultati relativi ai pazienti positivi a uno o più test specifici per la diagnosi di legionellosi.

Venticinque (pari al 6.1\%) dei 408 pazienti di cui sono stati analizzati i campioni d'urina per la ricerca dell'antigene specifico, sono risultati positivi: $6(8 \%)$ nel 2001, $11(6 \%)$ nel 2002 e $8(5 \%)$ nel 2003. In 2 pazienti sono stati invece riscontrati risultati dubbi su diversi campioni successivi esaminati.

La coltura dei 16 materiali provenienti dalle vie respiratorie ha portato all'isolamento di $L$. pneumophila in 5 casi: 4 nel 2001 (i campioni provenivano tutti da pazienti positivi all'antigenuria) e 1 nel 2002. In tutti si trattava di L. pneumophila sierotipo 1. Gli altri 6 materiali respiratori sottoposti ad esame colturale nel 2002 sono risultati tutti negativi: 2 campioni, prelevati da pazienti positivi alla ricerca dell'antigene specifico, vennero inviati rispettivamente a 4 e a 6 giorni di distanza dalla comunicazione della positività del test urinario, mentre le altre 4 colture eseguite riguardavano pazienti con quadri di insufficienza respiratoria, senza altri segni o sintomi suggestivi di legionellosi, nei quali la ricerca di Legionella spp. rientrava nell'ambito di un'ampia serie di indagini. In nessuno, infine, dei 4 campioni inviati nel 2003 la coltura ha fornito un risultato positivo: 3 materiali provenivano da pazienti in insufficienza respiratoria con antigenuria negativa, il quarto invece da un paziente risultato, il giorno precedente, positivo alla ricerca dell'antigene urinario.

Il numero di sieroconversioni, invece, si è mantenuto pressoché costante: 6 pazienti $(0.7 \%)$ nel 2001 e nel 2002, 6 pazienti (0.5\%) nel 2003. Considerando anche i dati relativi a titoli anticorpali significativi su singolo campione di sangue $(\geq 1: 256)$, la sieroprevalenza è risultata pari all'1.9\%.

In base alle indicazioni fornite dalle linee guida dell'ISS, le analisi eseguite presso il Servizio di Microbiologia dell'O.C.M. di Verona hanno definito 27 casi certi e 3 presunti di legionellosi, così ripartiti: 8 casi certi (di cui 7 comunitari e 1 nosocomiale) nel 2001 (tabella 1); 11 casi certi (di cui 1 di probabile origine nosocomiale) e 2 presunti (entrambi comunitari) nel 2002 (tabella 2); 8 casi certi e 1 presunto (tutti comunitari) nel 2003 (tabella 3).

Il $63 \%$ dei casi si è manifestato nei mesi compre- 
Tabella I. Caratteristiche microbiologiche dei casi di legionellosi verificatisi nel 200 I

\begin{tabular}{|c|c|c|c|c|c|}
\hline \multirow{2}{*}{ PAZIENTE } & \multirow[t]{2}{*}{ ANTIGENURIA } & \multirow{2}{*}{$\begin{array}{l}\text { COLTURA PER } \\
\text { LEGIONELLA SPP }\end{array}$} & \multicolumn{2}{|c|}{ TITOLAZIONE ANTICORPALE } & \multirow{2}{*}{$\begin{array}{c}\text { CLASSIFICAZIONE } \\
\text { DI CASO }\end{array}$} \\
\hline & & & I CAMPIONE & II CAMPIONE & \\
\hline $1 / 01$ & + & + & $1: 128$ & $1: 1024$ & certo \\
\hline $2 / 01$ & + & + & $<1: 128$ & I:2048 & certo \\
\hline $3 / 01$ & + & + & $<1: 128$ & N.R. & certo \\
\hline $4 / 01$ & + & + & $<1: 128$ & N.R. & certo \\
\hline $5 / 01$ & + & - & $<1: 128$ & $\mathrm{I}: 128$ & certo \\
\hline $6 / 01$ & N.R. & N.R. & $<\mathrm{I}: \mathrm{I} 28$ & $1: 512$ & certo \\
\hline $7 / 01$ & N.R. & N.R. & $1: 1024$ & $1: 4096$ & certo \\
\hline $8 / 01$ & N.R. & N.R. & $<1: 128$ & $1: 512$ & certo \\
\hline
\end{tabular}

+ : positivo; - : negativo; N. R. : non richiesto

atutti i ceppi isolati sono stati identificati come L. pneumophila I

'secondo le linee guida dell'ISS (I2)

Tabella 2. Caratteristiche microbiologiche dei casi di legionellosi verificatisi nel 2002

\begin{tabular}{|c|c|c|c|c|c|}
\hline \multirow[t]{2}{*}{ PAZIENTE } & \multirow[t]{2}{*}{ ANTIGENURIA } & \multirow{2}{*}{$\begin{array}{l}\text { COLTURA PER } \\
\text { LEGIONELLA SPP }\end{array}$} & \multicolumn{2}{|c|}{ TITOLAZIONE ANTICORPALE } & \multirow{2}{*}{$\begin{array}{c}\text { CLASSIFICAZIONE } \\
\text { DI CASO }\end{array}$} \\
\hline & & & I CAMPIONE & II CAMPIONE & \\
\hline 1102 & + & + & $<1: 128$ & N.R. & certo \\
\hline 2102 & + & N.R. & $<\mathrm{I}: \mathrm{I} 28$ & $1: 512$ & certo \\
\hline 3102 & + & - & $<\mathrm{I}: 128$ & $1: 256$ & certo \\
\hline 4102 & + & N.R. & $<\mathrm{I}: 128$ & 1:4096 & certo \\
\hline 5102 & + & N.R. & $1: 1024$ & $1: 4096$ & certo \\
\hline 6102 & + & - & $<1: 128$ & $1: 256$ & certo \\
\hline $7 \backslash 02$ & + & N.R. & $\mathrm{I}: 128$ & N.R. & certo \\
\hline 8102 & + & N.R. & $\mathrm{I}: 128$ & N.R. & certo \\
\hline 9102 & + & N.R. & $<1: 128$ & N.R. & certo \\
\hline 10102 & + & N.R. & $1: 1024$ & N.R. & certo \\
\hline 11102 & $+1-$ & N.R. & $<\mathrm{I}: \mathrm{I} 28$ & N.R. & presunto \\
\hline 12102 & $+1-$ & N.R. & $1: 256$ & N.R. & presunto \\
\hline 13102 & + & N.R. & N.R. & N.R. & certo \\
\hline
\end{tabular}

+ : positivo; - : negativo; +/ - : dubbio; N. R. : non richiesto

atutti i ceppi isolati sono stati identificati come L. pneumophila I

bsecondo le linee guida dell'ISS (I2)

Tabella 3. Caratteristiche microbiologiche dei casi di legionellosi verificatisi nel 2003

\begin{tabular}{|c|c|c|c|c|c|}
\hline \multirow[t]{2}{*}{ PAZIENTE } & \multirow[t]{2}{*}{ ANTIGENURIA } & \multirow{2}{*}{$\begin{array}{l}\text { COLTURA PER } \\
\text { LEGIONELLA SPP }\end{array}$} & \multicolumn{2}{|c|}{ TITOLAZIONE ANTICORPALE } & \multirow{2}{*}{$\begin{array}{c}\text { CLASSIFICAZIONE } \\
\text { DI CASO }\end{array}$} \\
\hline & & & I CAMPIONE & II CAMPIONE & \\
\hline II03 & + & N.R. & $<1: 128$ & $1: 512$ & certo \\
\hline 2103 & + & N.R. & $<1: 128$ & $1: 1024$ & certo \\
\hline 3103 & + & N.R. & $1: 128$ & N.R. & certo \\
\hline 4103 & + & N.R. & $\mathrm{I}: 256$ & N.R. & certo \\
\hline 5103 & + & N.R. & N.R. & N.R. & certo \\
\hline 6103 & + & N.R. & $<1: 128$ & N.R. & certo \\
\hline $7 \backslash 03$ & + & N.R. & $<1: 128$ & $1: 1024$ & certo \\
\hline 8103 & - & N.R. & $<1: 128$ & $1: 1024$ & presunto \\
\hline 9103 & + & & $<1: 128$ & $1: 512$ & certo \\
\hline
\end{tabular}

+ : positivo; - : negativo; N. R.: non richiesto

a tutti i ceppi isolati sono stati identificati come L. pneumophila I

b secondo le linee guida dell'ISS (I2)

si tra agosto e ottobre. Per quanto riguarda le caratteristiche dei pazienti, l'età media è risultata pari a 53 anni, con un range compreso tra 26 e 75 anni e il sesso maschile è risultato nettamente più colpito (83\% dei casi osservati) con un rapporto maschi/femmine pari a $5: 1$. Il $53 \%$ dei pazienti era fumatore, il $14 \%$ etilista, il $10 \%$ presentava patologie predisponenti (malattie neoplastiche e interventi di trapianto) mentre nel $21 \%$ dei casi osservati la sintomatologia comparve dopo un soggiorno fuori casa.

Dal punto di vista clinico tutti i pazienti, tranne uno, presentarono un quadro di polmonite con febbre (nel $100 \%$ dei casi), tosse (43\%) e dispnea
(18\%); 3 pazienti (11\%) accusarono anche alterazioni neurologiche (cefalea e obnubilamento del sensorio) e 6 pazienti (21\%) disturbi gastrointestinali. Uno dei pazienti osservati nel 2002 presentò esclusivamente un quadro di miocardite senza coinvolgimento polmonare.

Il $48 \%$ dei pazienti è stato trattato con claritromicina o levofloxacina in monoterapia mentre il $37 \%$ con l'associazione dei due. Per 2 pazienti $(4 / 01,1 / 02)$ l'esito della malattia fu infausto.

\section{DISCUSSIONE}

Le caratteristiche epidemiologiche dei casi riportati nel nostro studio (stagionalità, distribuzione 
rispetto all'età e al sesso, fattori di rischio) sono sostanzialmente in linea con i dati riportati in letteratura $(1,14,15)$, come pure sono già stati descritti rari casi di miocardite e pericardite da $L$. pneumophila in assenza di polmonite $(5,12)$.

Gli aspetti che si vogliono sottolineare in questo lavoro riguardano invece il cambiamento che l'introduzione della ricerca dell' antigene urinario ha indotto nell'approccio diagnostico di questi quadri morbosi e le conseguenze osservate, nonché i limiti della stessa se condotta come test isolato.

Nel corso dei 3 anni, questo tipo di indagine è diventato lo strumento diagnostico maggiormente richiesto a scapito di altri, primo fra tutti l'esame colturale. Se infatti nel 2001 è stato possibile eseguire la coltura nel 7\% dei casi sospetti, intendendo tali i pazienti per i quali venne richiesta la ricerca dell'antigene urinario, nel 2002 tale percentuale è scesa al $4 \%$ e al $2.5 \%$ nel 2003 ; nonostante l'esiguo numero di casi analizzati inoltre, è emersa chiaramente la difficoltà ad ottenere i materiali idonei all'esame colturale nei tempi utili per l'isolamento del microrganismo.

Come già riportato in letteratura $(11,15,13,17,8$, 2) se da un lato la valutazione dell'antigenuria, per la semplicità di esecuzione e la rapidità con cui fornisce il risultato, ha significativamente migliorato le capacità diagnostiche nei confronti delle infezioni sostenute da $L$. pneumophila con evidenti e importanti ripercussioni anche in ambito di sanità pubblica, dall'altro il suo utilizzo in maniera esclusiva ha conseguenze indubbiamente negative.

Il mancato isolamento del germe infatti pregiudica la possibilità di condurre corrette indagini epidemiologiche, di confrontare i ceppi di isolamento clinico con quelli ambientali e di conoscere la distribuzione e il significato clinico di specie e sierogruppi diversi da L. pneumophila 1 ; come è noto, infatti, i prodotti commercializzati per la ricerca dell'antigene urinario sono validati esclusivamente per le infezioni sostenute da questo sierotipo, nonostante per alcuni venga dichiarata una sensibilità variabile anche nei confronti di sierotipi diversi $(11,13,15)$. A questo proposito in uno dei casi osservati (8\03: tabella 3 ) alla negatività della ricerca dell'antigene urinario specifico (non fu richiesta purtroppo la coltura) è seguita una sieroconversione nei confronti del pool costituito da L. pneumophila sierotipi 10, 12, 14 .

In altri 2 casi inoltre (11/02 e 12/02: tabella 2$)$, la ricerca dell'antigene urinario eseguita su campioni successivi non ha fornito risultati chiaramente interpretabili e l'impossibilità di eseguire indagini colturali così come valutazioni sierologiche specifiche su doppio campione non ha permesso di giungere ad una diagnosi eziologica malgrado entrambi i pazienti presentassero un quadro clinico di polmonite.

Alla luce di quanto osservato in questi 3 anni riteniamo che sia limitante, ed in alcuni casi anche fuorviante, utilizzare la valutazione dell'antigenuria come unico approccio microbiologico nella diagnosi delle infezioni sostenute da Legionella spp., anche se, come indicato nelle linee guida dell'ISS, la sola positività di questo test è considerata sufficiente a definire con certezza un caso. Se nel 2001 1' $80 \%$ dei casi positivi alla ricerca dell' antigene urinario è stato confermato attraverso indagini di altro tipo (colturali o sierologiche), nel 2002 e nel 2003 tale percentuale è scesa rispettivamente al $54 \%$ e al $56 \%$. Il solo approccio diagnostico rappresentato dal test urinario non ha permesso la corretta valutazione di 2 casi osservati nel $2002(11 / 02$ e 12/02) mentre il saggio sierologico eseguito su doppio campione ha individuato un caso di falsa negatività del test urinario, fra i pazienti analizzati nel 2003.

Pur riconoscendo l'importanza della ricerca dell'antigene urinario quale strumento di diagnosi rapida, riteniamo che questo test debba sempre essere affiancato all'indagine colturale e sierologica sia per confermare i risultati positivi dell'antigenuria (non sempre inequivocabili) sia per individuare infezioni sostenute da specie e sierotipi diversi, la cui epidemiologia rimane, altrimenti, del tutto oscura.

\section{BIBLIOGRAFIA}

1. Andreoni G, Bonazzi L, et al. Clinical epidemiological survey of Legionella pneumophila in Italy. J Int Med Res 1986; 14(6): 325-9.

2. Benin AL, Benson RF, Besser RE. Trends in legionnaires' disease, 1980-1998: declining mortality and new patterns of diagnosis. Clin Infect Dis 2002; 35: 1039-46.

3. Benson RF, Fields BS. Classification of the genus Legionella. Semin Respir Infect 1998; 13: 90-9.

4. Castellani Pastoris M, Nigro G, Midulla M. Arrhythmia or myocarditis: a novel clinical form of Legionella pneumophila infection in children without pneumonia. Eur J Pediatr 1985; 144: 157-9

5. Edelstein PH. Legionnaires' disease. Clin Infect Dis 1993; 16: 741-9.

6. Helbig J, Uldum S, et al. Clinical utility of urinary antigen detection for diagnosis of communityacquired, travel-associated, and nosocomial legionnaires' disease. J Clin Microbiol 2003; 41 (2): 838-40.

7. Fields BS, Benson RF, Besser E. Legionella and legionnaires' disease: 25 years of investigation. Clin Microbiol Rev 2002; 15(3): 506-26.

8. Istituto Superiore di Sanità Linee guida per la prevenzione ed il controllo della legionellosi. Gazzetta Ufficiale 05/05/2000.

9. Yu VL. Legionella pneumophila (legionnaires' disease). In: Priciples and practice of infectious diseases, Mandell G L,Bennett J E, Dolin R, Churchill 
Livingstone Inc, New York 1995; 2087-97.

10. Yzerman EPF, den Boer J W, et al. Sensitivity of three urinary antigen tests associated with clinical severity in a large outbreak of legionnaires' disease in the Netherlands 2002; 40(9): 3232-6.

11. Nelson DP, Rensimer ER, Raffin TA. Legionella pneumophila pericarditis without pneumonia. Arch Intern Med 1985; 145: 926.

12. Mandell GL, Bennett JE, Dolin R. Priciples and practice of infectious diseases $4^{\text {th }}$ ed. Cap. 211

13. Murdoch D R. Diagnosis of Legionella infection. Clin Infect Dis 2003; 36: 64-9.
14. Waterer W, Baselski V, et al. Legionella and community acquired pneumonia: a review of current diagnostic tests from a clinician's viewpoint. The American Journal of Medicine 2001; 110: 41-8

15. Roig J, Rello J. Legionnaires' disease: a rational approach to therapy. Journal of Antimicrobial Chemotherapy 2003; 51: 1119-29.

16. Ruehlemann SA, Crawford GR. Panic in the pottig shed: the association between Legionella longbeachae serogroup 1 and potting soils in Australia. Med J Aust 1996; 164: 36-8.

\section{Manuela Pegoraro}

Servizio di Microbiologia, Immunologia e Virologia, Ospedale Civile Maggiore,

P.le Stefani 1, 37126 Verona

Tel.: 045 8072461; Fax 0458073304

E-mail: manuela.pegoraro@azosp.vr.it 